\title{
Mesoscopic Hamiltonian for the fluctuations of adsorbed Lennard-Jones liquid films
}

\author{
Eva M. Fernández* \\ Departamento de Física Fundamental, Universidad Nacional de Educación Distancia, Madrid, Spain \\ and Instituto de Ciencia de Materiales de Madrid, CSIC, 28049, Madrid, Spain \\ Enrique Chacón ${ }^{\dagger}$ \\ Instituto de Ciencia de Materiales de Madrid, CSIC, 28049, Madrid, Spain \\ and Instituto de Ciencia de Materiales Nicolás Cabrera, Universidad Autońoma de Madrid, Madrid, 28049, Spain \\ Luis G. MacDowell \\ Departamento de Química Física, Universidad Complutense de Madrid, 28040, Madrid, Spain \\ Pedro Tarazona ${ }^{\S}$ \\ Departamento de Física Teórica de la Materia Condensada, Condensed Matter Physics Center IFIMAC and Instituto de Ciencia de \\ Materiales Nicolás Cabrera, Universidad Autónoma de Madrid, Madrid, 28049, Spain
}

(Received 13 March 2015; published 19 June 2015)

\begin{abstract}
We use Monte Carlo simulations of a Lennard-Jones fluid adsorbed on a short-range planar wall substrate to study the fluctuations in the thickness of the wetting layer, and we get a quantitative and consistent characterization of their mesoscopic Hamiltonian, $\mathcal{H}[\xi]$. We have observed important finite-size effects, which were hampering the analysis of previous results obtained with smaller systems. The results presented here support an appealing simple functional form for $\mathcal{H}[\xi]$, close but not exactly equal to the theoretical nonlocal proposal made on the basis a generic density-functional analysis by Parry and coworkers. We have analyzed systems under different wetting conditions, as a proof of principle for a method that provides a quantitative bridge between the molecular interactions and the phenomenology of wetting films at mesoscopic scales.
\end{abstract}

DOI: 10.1103/PhysRevE.91.062404

PACS number(s): 68.08.Bc, 68.03.Kn, 68.03.Hj

\section{INTRODUCTION}

Liquid films adsorbed on solid substrates are important in nature and they have a great number of practical applications $[1,2]$. Their equilibrium mean thickness $\bar{\xi}$ increases when the vapor approaches saturation and, for systems above the wetting temperature, the film becomes macroscopically thick at bulk liquid-vapor coexistence. Mean-field theories [3] predict $\bar{\xi}$ in terms of an effective potential, $\Phi(\bar{\xi})=\Delta \Omega_{\text {exc }}(\bar{\xi}) / A_{0}$, which gives the thermodynamic grand-potential excess per unit area, with respect to the thick liquid film.

At mesoscopic scale, thick adsorbed films develop strong fluctuations of their local thickness $\xi(\mathbf{R})$, with $\mathbf{R}=(x, y)$ on the substrate plane, which are important for many applications. Their theoretical description [4] is based on effective Hamiltonians $\mathcal{H}[\xi]$, modeled as functionals of $\xi(\mathbf{R})$, following the capillary wave theory (CWT) for free liquid surfaces [5-8] or from simple density-functional models [9]. In this paper we present a fully quantitative description of $\mathcal{H}[\xi]$ for a realistic model: a Lennard-Jones fluid adsorbed on a planar wall, analyzed with the intrinsic sampling method (ISM) for the shape of a fluctuating fluid interface [10,11]. Our results provide a proof of principle for the method to bridge the gap between computer simulations at molecular scale and the mesoscopic behavior of these systems with quantitative accuracy.

\footnotetext{
*emfernandez@fisfun.uned.es

†echacon@icmm.csic.es

${ }^{\ddagger}$ lgmac@quim.ucm.es

§pedro.tarazona@uam.es
}

The instantaneous fluctuations of the film thickness around its mean value $\bar{\xi}$, calculated over the whole substrate area $A_{0}$, are described as $\xi(\mathbf{R})=\sum_{q} \hat{\xi}_{q} \exp (i \mathbf{q} \cdot \mathbf{R})$ in terms of its Fourier components $\mathbf{q}=\left(q_{x}, q_{y}\right)$. Assuming a perfectly flat substrate, at temperature $k_{\mathrm{b}} T \equiv \beta^{-1}$, the thermal fluctuations give mean values $\left\langle\hat{\xi}_{q}\right\rangle=0$ for the $q \neq 0$ Fourier components, and the mesoscopic Hamiltonian expanded at order $\left|\hat{\xi}_{q}\right|^{2}$,

$$
\mathcal{H}[\xi(\mathbf{R})]=A_{0} \Phi(\bar{\xi})+\frac{1}{2 \beta} \sum_{q} \Gamma(q, \bar{\xi})\left|\hat{\xi}_{q}\right|^{2}+\mathcal{O}^{4}\left(\hat{\xi}_{q}\right),
$$

has a spectrum of harmonic modes fully described by $\Gamma(q, \bar{\xi}) \equiv 1 /\left\langle\left|\hat{\xi}_{q}\right|^{2}\right\rangle$, the (inverse) mean square amplitude for each $q$ and as a function of the mean film thickness $\bar{\xi}$. The classical theory takes $\Gamma_{\text {class }}(q, \bar{\xi}) \approx \beta A_{0}\left(\Phi^{\prime \prime}(\bar{\xi})+q^{2} \gamma_{\mathrm{LV}}\right)$, from the Hamiltonian

$$
\mathcal{H}_{\text {class }}[\xi(\mathbf{R})]=\int d^{2} \mathbf{R}\left\{\Phi[\xi(\mathbf{R})]+\frac{\gamma_{\mathrm{LV}}}{2}|\nabla \xi(\mathbf{R})|^{2}\right\},
$$

where the first term describes the interaction of the wetting film with the inert substrate, in terms of the local effective potential $\Phi(\bar{\xi})$, and the second term considers the corrugations of the film, as a free liquid-vapor surface with surface tension $\gamma_{\mathrm{LV}}$ that multiplies the (instantaneous) increase of the area produced by the fluctuations at the edge of the film. Curvature, $\left(\nabla^{2} \xi\right)^{2}$, and higher-order terms are neglected.

There has been some controversy $[9,12]$ over the limitations of Eq. (2). It could be expected that for thin films the surface tension $\gamma_{\mathrm{LV}}$ in Eq. (2) should depend on the film thickness, so that $\Gamma(q, \bar{\xi}) \approx \beta A_{0}\left\{\Phi^{\prime \prime}(\bar{\xi})+q^{2}\left[\gamma_{\mathrm{LV}}+\Delta \gamma(\bar{\xi})\right]\right\}$. Moreover, even for very large $\bar{\xi}$ [when both $\Phi^{\prime \prime}(\bar{\xi})$ and $\Delta \gamma(\bar{\xi})$ vanish], the free liquid surface is expected to have a bending energy term, 
which may be described as a wave-vector-dependent function $\gamma^{\mathrm{LV}}(q)=\gamma_{\mathrm{LV}}+\kappa_{\mathrm{LV}} q^{2}+\cdots$.

In general, we may write

$$
\frac{\Delta \Gamma(q, \bar{\xi})}{\beta A_{0}} \equiv \frac{\Gamma(q, \bar{\xi})}{\beta A_{0}}-q^{2} \gamma^{\mathrm{LV}}(q)=\Phi^{\prime \prime}(\bar{\xi})+q^{2} \Delta \gamma(q, \bar{\xi}),
$$

where the function $\Delta \gamma(q, \bar{\xi})$ contains together all the effects beyond the local evaluation of $\Phi[\xi(\mathbf{R})]$, in the first term of Eq. (2) and all the thickness and wavevector dependence of the effective surface tension at the edge of the film, beyond the free liquid surface $\gamma^{\mathrm{LV}}(q)$.

Within the generic form, Eq. (3), the nonlocal Hamiltonian form used by Fisher and Jin [13] corresponds to neglect of the $q$ dependence, $\Delta \gamma(\bar{\xi}, q) \approx \Delta \gamma_{0}(\bar{\xi})$, and $\gamma^{\mathrm{LV}}(q) \approx \gamma_{\mathrm{LV}}$, so that $\Gamma_{\mathrm{FJ}}(q, \bar{\xi})=\Phi^{\prime \prime}(\bar{\xi})+q^{2}\left[\gamma_{\mathrm{LV}}+\Delta \gamma_{\mathrm{FJ}}(\bar{\xi})\right]$. In contrast, the fully nonlocal theory of Parry et al. [14] includes the $q$ dependence, both in $\Delta \gamma(q, \bar{\xi})$ and in $\gamma^{\mathrm{LV}}(q)$, as it comes out of the linear response in a generic density-functional treatment $[9,12]$.

The experimental validation [15-17] of mesoscopic Hamiltonian models has to discriminate these subtle effects from the simple local Hamiltonian Eq. (2). A promising alternative is to use computer simulations that provide full information on the film structure at molecular level. In recent years there has been an increase in the studies based on simulations in order to confirm the nonlocal effects [18-20]; e.g., Pang et al. [18] present Monte Carlo (MC) simulations in Ising models and analyze various correlation functions of interface showing the first evidences of nonlocal effects. The development of approaches to sample the intrinsic surface, which represents the instantaneous molecular frontier between the liquid and the vapor phases [7], has opened a link between the mesoscopic effective Hamiltonians and computer simulations for realistic fluid models. Nonlocal effects have been found in simulations of the free liquid surface for the Lennard-Jones (LJ) model [21], with a correlation between the intrinsic profile and the interfacial position that matches precisely the predictions of the nonlocal theory $[9,12]$.

Recently, we have applied the intrinsic sampling method [22] to get directly $\Phi(\xi)$ and $\Gamma(q, \xi) \equiv\left\langle\left|\hat{\xi}_{q}\right|^{2}\right\rangle^{-1}$ from computer simulation for liquid films, with (truncated) LJ interactions, adsorbed on a planar wall. The system was deep in the total wetting regime, so that the effective potential $\Phi(\xi)$ had a large negative slope, pushing the film toward the equilibrium thick liquid slab. The computer simulation results, for chemical potential at liquid-vapor coexistence, gave an excellent fit to the pure exponential form,

$$
\Phi(\bar{\xi})=\Phi_{0} \exp (-\lambda \bar{\xi}),
$$

with the amplitude $\Phi_{0}$ as the only fitting parameter, since the inverse decay length $\lambda$ was observed to be the same as in the asymptotic decay of the density profile at the free liquidvapor interface [23]. This is in agreement with the densityfunctional analysis for any system with truncated or shortrange interactions. Only for long-ranged interactions between the fluid molecules [24,25] or between the wall and the fluid $[26,27]$ we expect that the asymptotic functional form of $\Phi(\bar{\xi})$ deviates from Eq. (4), but still it was surprising to observe that, in a realistic model with strong layering effects in the density profiles, the pure exponential decay could give such accurate representation of $\Phi(\bar{\xi})$, even for very thin films of just one or two molecular layers.

In this paper, we present simulations closer and beyond the wetting transition, i.e., reducing the value of $\Phi_{0}$ to small positive and negative values. From the theoretical predictions of Parry et al. [9,12], from a simplified density-functional model, $\Phi(\bar{\xi})$ should contain a second exponential term $\sim e^{-2 \lambda \bar{\xi}}$, and the comparison of systems at different wetting condition could discern if the apparent lack of that term in our simulation results was generic or fortuitous. To get a clean view of the wall effects, we keep exactly the same LJ model (truncated at a cutoff distance $2.5 \sigma$ ) and temperature, $k_{\mathrm{b}} T / \epsilon=0.75$, in the usual $\sigma$ and $\epsilon$ parameters of the LJ model. The inverse decay length $\lambda=1.55 / \sigma$, in Eq. (4) is fixed by the liquid bulk phase. To change the wetting regime we tune the strength of the wall-fluid interaction, relative to $\epsilon$ in the fluid, with the parameter $\epsilon_{\mathrm{sf}} / \epsilon$ [see, e.g., Eq. (9) in Ref. [22]], which we reduce from $\epsilon_{\mathrm{sf}} / \epsilon=1.3$ (used in our previous simulations) to 0.81 and 0.77 , just above and below the wetting transition, that we locate at $\epsilon_{\mathrm{sf}} / \epsilon=0.785 \pm 0.005$. We refer the reader to the Appendix and to our previous paper [22] for the details on the model, computer simulation, and the methods to obtain $\Phi(\xi)$ and $\Gamma(q, \xi)$, from the fluctuations of the film.

The other relevant change is the size of the simulated systems, with a ninefold increase of the substrate area and up to $N=6750$ molecules in the thickest films. The motivation for this size increase was the strongly oscillatory structure found in our results for $\Delta \Gamma(q, \bar{\xi})$, compared with the simple exponential decay observed in $\Phi(\bar{\xi})$. As we had anticipated, the layering structure observed in $\Delta \Gamma(q, \bar{\xi})$ was indeed a size effect. The computational effort required to simulate large systems over long times, to sample the slow fluctuations with low $q$, has shown to be necessary to extract the correct form for the mesoscopic Hamiltonian Eq. (1). The Appendix presents the details of the model, system sizes, and methods of simulation and analysis.

\section{THE EFFECTIVE WALL POTENTIAL}

We have used grand-canonical Monte Carlo simulations, with a shifting constraint in the total number of particles, to get the relative probabilities for film thickness up to $5 \sigma$. The procedure, following the previous works of MacDowell and Müller [28,29], was explained in our previous paper [23], with two alternative method to determine the mean thickness of the wetting film (see Appendix). The simplest is the Gibbs' dividing (GD) plane, with $\bar{\xi}=\xi_{N}$ determined by the instantaneous number of particles, the second estimation of the mean thickness, $\bar{\xi}=\xi_{\text {IS }}$, is based on the intrinsic surface (IS) at the edge of the layer, as given by the ISM with a percolation analysis and the identification of the outermost liquid layer.

The top panel of Fig. 1 presents the effective wall potentials $\Phi\left(\xi_{N}\right)$ and $\Phi\left(\xi_{\text {IS }}\right)$ for $\epsilon_{\text {sf }} / \epsilon=0.81$, taking $\Phi(\xi)=0$ for thick film at bulk coexistence. Both methods give very similar effective potentials and they exhibit a clear exponential behavior [Eq. (4)] from the submonolayer range to the limit of our resolution. The only difference with our previous result is the change in $\Phi_{0}\left(\epsilon_{\mathrm{sf}} / \epsilon\right)$, from $\beta \Phi_{0}(1.3) \sigma^{2}=1.97$ 


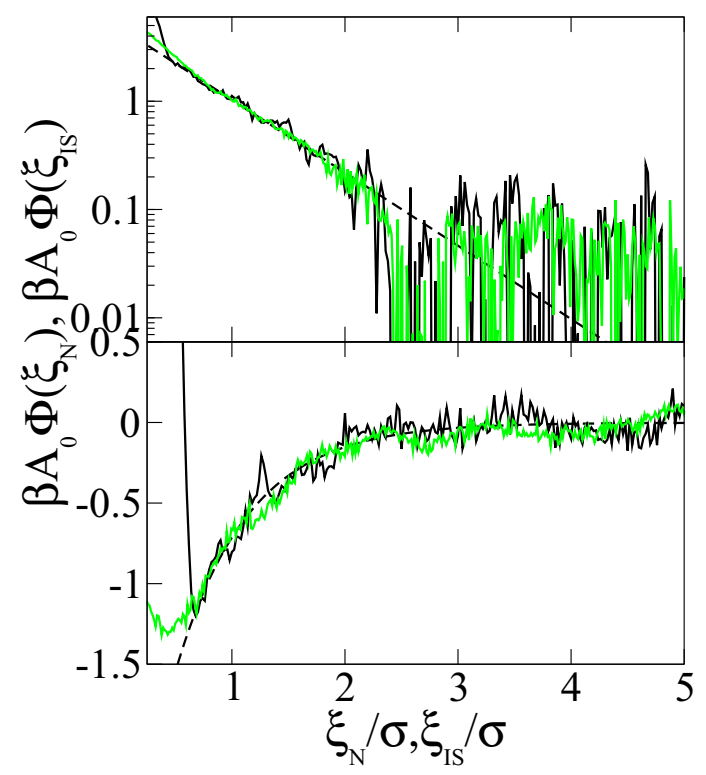

FIG. 1. (Color online) Interfacial potential $\Phi(\bar{\xi})$ as a function of the mean thickness of the liquid film, for two strengths of the wall-fluid attraction: $\epsilon_{\mathrm{sf}} / \epsilon=0.81$ (top) and 0.77 (bottom). The light (green) curves use $\bar{\xi}=\xi_{N}$, from the total number of particles. The dark (black) curves use $\bar{\xi}=\xi_{\text {IS }}$ from the ISM position of the film edge. The dashed lines show the exponential fits [Eq. (4) with $\lambda=1.55 / \sigma$ ] to $\Phi\left(\xi_{\text {IS }}\right)$.

to $\beta \Phi_{0}(0.81) \sigma^{2}=0.049$, as a signal of the approach to the complete wetting regime. Notice that Fig. 1 presents $\beta \Phi(\bar{\xi}) A_{0}$, i.e., the total grand-potential change associated to the change of the film thickness over the entire system with $A_{0}=100 \sigma^{2}$. The equilibrium configuration, at liquid-vapor bulk coexistence, would be a very thick (macroscopic) liquid layer, but a fluctuation that reduces the film to a monolayer $(\bar{\xi} \approx \sigma)$ in our simulation box would require only an increase of $\beta \Delta \Omega \approx 1$, making it a very probable event. In contrast, with $\epsilon_{\mathrm{sf}} / \epsilon=1.3$, that fluctuation would imply $\beta \Delta \Omega \approx 40$, i.e., it would be impossible to sample it in unconstrained grand-canonical simulations.

The bottom panel in Fig. 1 shows (in linear scale) the effective potential for $\epsilon_{\mathrm{sf}} / \epsilon=0.77$. As expected for the partial wetting regime, $\Phi(\bar{\xi})$ shows a minimum with $\beta \Phi(\bar{\xi}) A_{0} \approx$ -1.3 . The tail of $\Phi(\bar{\xi})$ is still well fitted by a pure exponential form [Eq. (4)], with $\lambda=1.55 / \sigma$ and $\beta \Phi_{0}(0.77) \sigma^{2}=-0.034$, and it is very similar for the two alternative definitions for the mean thickness of the liquid film. In the submonolayer regime the ISM breaks down, with a sharper increase in $\Phi(\xi)$, since the adsorbed film does not percolate over the substrate plane. Using the results for the minimum of $\Phi\left(\xi_{N}\right)$, the macroscopic contact angle may be $\theta=\cos ^{-1}\left(1+\Phi_{\min } / \gamma_{\mathrm{LV}}\right) \approx 0.2 \mathrm{rad}$; i.e., in a macroscopic system any liquid film with $\xi \lesssim 1 \sigma$ would be unstable (or at most metastable). A flat liquid drop would form on the substrate, with contact angle of just 11 degrees. In our simulations, even with the larger transverse size $L_{x}=30 \sigma$, the formation of those drops is frustrated by the periodic boundary conditions, and we may sample these (finite size-stabilized) liquid films to get $\Phi(\bar{\xi})$, from their relative stability in the grand-canonical ensemble, and $\Gamma(q, \bar{\xi})$ from their fluctuations in canonical ensemble Monte Carlo simulations.
The main conclusion of these results is that the simple exponential decay Eq. (4), with the inverse correlation length of the bulk liquid, describes very accurately the effective solidfilm potential, for films thicker than a molecular monolayer. All the details of the solid-fluid interaction are reduced to the value of $\Phi_{0}\left(\epsilon_{\mathrm{sf}} / \epsilon\right)$, which may be observed to become negative as the system goes through the wetting transition. There is no signature of the $\sim e^{-2 \lambda \xi}$ term predicted by the theoretical analysis for the Landau-Ginsburg-Wilson density-functional model, within the double parabola approximation [9]. Of course, in the partial wetting regime the minimum of $\Phi(\bar{\xi})$ requires that a short-range repulsion stops the tendency of Eq. (4) with $\Phi_{0}<0$, but its effects are limited to films in the submonolayer range and they are determined by the form of the short-range solid-fluid repulsion, rather than by the liquid bulk correlation length. From the theoretical side, the predictions of the simplified density-functional models should be understood as the bare binding potential for the interface, while our simulation results should give a partially renormalized version, dressed by the fluctuations allowed by the simulation box size, which could transform the bare $\exp (-2 \lambda \bar{\xi})$ term into a renormalized Gaussian even more difficult to observe.

Finally, the wetting transition of our system is expected to be of first order. In a mean-field description the effective potential would exhibit a barrier between the thin-film minimum and the macroscopic wetting layer [4]. In a computer simulation that barrier would be reduced with increasing size, as the correlations are included up to larger distances, and the accuracy of our results does not allow to resolve any signature of it. Notice, in this respect, that (as for the $\epsilon_{\text {sf }} / \epsilon=0.81$ case) Fig. 1 presents $\beta \Phi(\xi) A_{0}$. The fluctuations of the film thickness over the whole substrate area $A_{0}=100 \sigma^{2}$ could take it easily from the thin-film minimum to a very thick one.

\section{CW FLUCTUATIONS AT THE EDGE OF WETTING LAYERS}

We have run canonical ensemble Monte Carlo simulations, for the three values of $\epsilon_{\mathrm{sf}}$ studied in this work and the two different cells sizes. The number of particles was chosen to sample the relevant mean thickness of the adsorbed liquid film. We present here ISM results, based on the surface of the percolating cluster, and in the Appendix we compare them with the simpler (but less accurate) representation given by the Gibbs dividing surface. For each $\xi_{\text {IS }}$, we get the mean-square fluctuations $\left\langle\left|\hat{\xi}_{q}\right|^{2}\right\rangle$ for all the sampled wavevectors: $q \sigma=$ $0.21,0.6,0.85,1.22$, and 1.9 in the larger simulation boxes, while $q \sigma=0.6$ is the smallest value accessible in the smaller boxes. The ISM analysis for the free liquid-vapor interface, with the same box sizes, was used to obtain $\gamma^{\mathrm{LV}}(q)$ and, from Eq. (3), the wall damping effect $\Delta \Gamma\left(q, \xi_{\text {IS }}\right)$ presented in Fig. 2 for $q \sigma=0.6$, as functions of the film thickness $\xi_{\text {IS }}$. For other values of $q$ we observe similar shapes but different amplitudes, as presented in Fig. 3 for $\epsilon_{\mathrm{sf}} / \epsilon=0.81$, with the larger box size.

The first interesting result is that, comparing the two first columns in Fig. 2, we can address the question about the oscillatory behavior of the $\mathrm{CW}$ damping. The behavior was reported in our previous work, and it is reproduced here in the simulations for lower $\epsilon_{\mathrm{sf}}$ but the same simulation box size. Less attractive walls $\left(\epsilon_{\mathrm{sf}} / \epsilon=0.81\right.$ and 0.77$)$ produce 


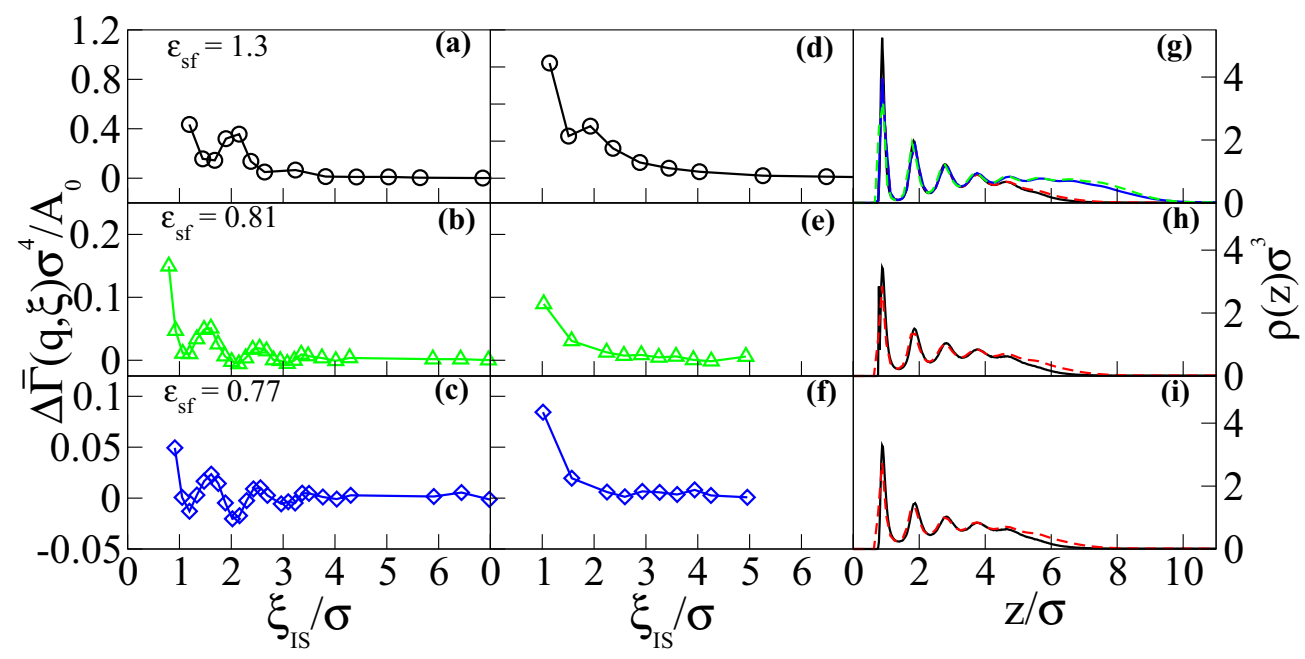

FIG. 2. (Color online) Wall damping effect on the inverse mean-square amplitude of the thickness fluctuations, $\Delta \Gamma\left(q\right.$, $\left.\xi_{\mathrm{IS}}\right)$, Eq. (3), for the wave number $q \sigma=0.6$, with increasing thickness of the adsorbed liquid film, for the three values of the wall fluid attraction, $\epsilon_{\mathrm{sf}} / \epsilon=1.3$ (top), 0.81 (central), and 0.77 (bottom); and two lateral sizes of the simulation box: $L_{x}=10.3 \sigma$ (left) and $L_{x}=31 \sigma$ (central). Density profile (right) of $\xi_{\text {IS }} \simeq 4.40 \sigma$ (dark color) and $\xi_{\text {IS }} \simeq 7.72 \sigma$ (light color) films for $L_{x} \simeq 10.3 \sigma$ (solid line) and $\xi_{\text {IS }} \simeq 4.80 \sigma$ (dark color) and $\xi_{\text {IS }} \simeq 8.10 \sigma$ (light color) for $L_{x} \simeq 31 \sigma$ (dashed line).

weaker oscillations in the density profiles than in the previous results for $\epsilon_{\mathrm{sf}} / \epsilon=1.3$, but relatively stronger oscillations in $\Delta \Gamma\left(q, \xi_{\text {IS }}\right)$, because the strong reduction in the value of $\Phi_{0}$ near the wetting transition makes the oscillations appear clearer over the monotonic term $\Phi^{\prime \prime}\left(\xi_{\text {IS }}\right)=\lambda^{2} \Phi_{0} e^{-\lambda \xi_{\text {IS }}}$ in Eq. (3). However, the oscillations are strongly damped in the $\Delta \Gamma\left(q, \xi_{\text {IS }}\right)$ results with a substrate area nine times larger than in the original size. Notice that the density profiles are essentially unaffected by the increase of the system's size (Fig. 2). Only for very thick films the larger size is reflected in a slightly broader edge of the liquid film. Therefore, that oscillatory structure in $\Delta \Gamma\left(q, \xi_{\text {IS }}\right)$ and $\Delta \gamma\left(q, \xi_{\text {IS }}\right)$ should not be considered as a real characteristics of the effective Hamiltonian $\mathcal{H}[\xi]$ Eq. (1), but as a finite-size effect that produces a pseudolayering transition when the lateral size of the system is not large enough. We had already hinted to that explanation [22] as we observed that

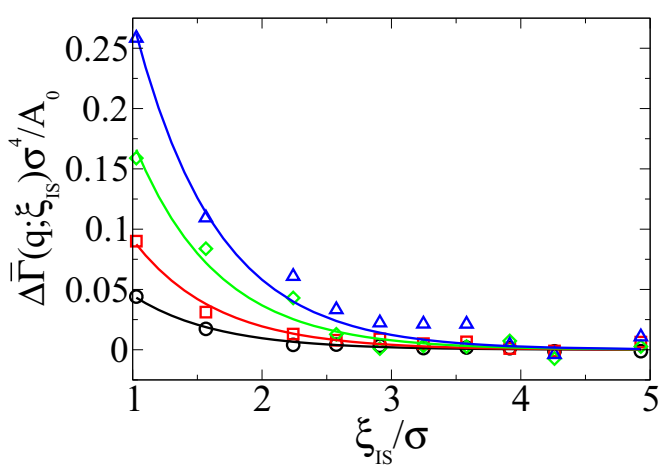

FIG. 3. (Color online) Wall-damping effect on inverse meansquare amplitude of the film-thickness fluctuation, $\Delta \Gamma\left(q, \xi_{I S}\right)$ with increasing thickness of the adsorbed liquid film, for $\epsilon_{\mathrm{sf}} / \epsilon=0.81$ and larger lateral size $L_{x}=31 \sigma$. Circles, $q \sigma=0.21$; squares, $q \sigma=0.6$; diamonds, $q \sigma=0.85$; and triangles up, $q \sigma=1.22$. The full lines are the best fits to an exponential decay $\Delta \Gamma\left(q, \xi_{\mathrm{IS}}\right)=$ $\Delta \Gamma_{0}(q) \exp \left(-1.55 \xi_{\mathrm{IS}} / \sigma\right)$ for each $q$. the upper envelop of the oscillatory structure showed the same simple exponential decay as $\Phi\left(\xi_{\text {IS }}\right)$.

The results for all the wall attractions are very close to the pure exponential decay $\Delta \Gamma\left(q, \xi_{\text {IS }}\right) \approx \Delta \Gamma_{0}(q) e^{-\lambda \xi_{\text {IS }}}$, with an amplitude that depends on $q$, Fig. 3 , and is very close to the upper envelop of the oscillatory structure obtained with the smaller box size. Therefore, we may conclude that a single exponential decay, as in Eq. (4), with the bulk correlation length of the liquid, describes accurately the whole dependence of the inverse mean-square fluctuations on the mean film thickness. Only for $\epsilon_{\mathrm{sf}} / \epsilon=1.3$ the exponential behavior is broken, at $\xi_{\text {IS }} \lesssim 2.0 \sigma$, probably as the result of the very strong wall-fluid interaction, which produces a very structured first layer. For the highest $q$ the results for $\epsilon_{\mathrm{sf}} / \epsilon=0.81$ seem also to show some slight oscillations; however, these fluctuations are clearly below the accuracy of our procedure.

The values for $\Delta \Gamma_{0}(q)$ that give the best fits to the exponential decay, and the error bars given by the dispersion of $\Delta \Gamma_{0}(q) e^{\lambda \xi_{\mathrm{IS}}}$, over the first five values of $\xi_{\mathrm{IS}}$, are presented in Fig. 4 to show the dependence on the wavevector. According to Eq. (3), and once we have observed that all the dependence of $\mathcal{H}([\xi])$ with the mean film thickness is well represented by the same exponential form, Eq. (4), we should have

$$
\frac{\Delta \Gamma_{0}(q, \xi)}{\beta A_{0}} \approx\left[\lambda^{2} \Phi_{0}+q^{2} \Delta \gamma_{0}(q)\right] e^{-\lambda \xi_{\mathrm{IS}}} .
$$

The $q \neq 0$ fluctuations, observed in the canonical ensemble simulations, may be extrapolated to $q=0$ where $\Delta \gamma_{0}(q)$ becomes irrelevant, and $\Delta \Gamma(0, \bar{\xi})=\beta A_{0} \Phi^{\prime \prime}(\bar{\xi})=\beta A_{0} \lambda^{2} \Phi_{0}$, is given by our independent result $\Phi(\bar{\xi})$, from the relative probabilities of each mean thickness from our grand-canonical simulations. This consistency test is passed for the three values of $\epsilon_{\mathrm{sf}}$. Our results for $\lambda^{2} \Phi_{0}$ (filled symbols in Fig. 4) are in good agreement with the extrapolation to $q=0$ of the respective open symbols. 


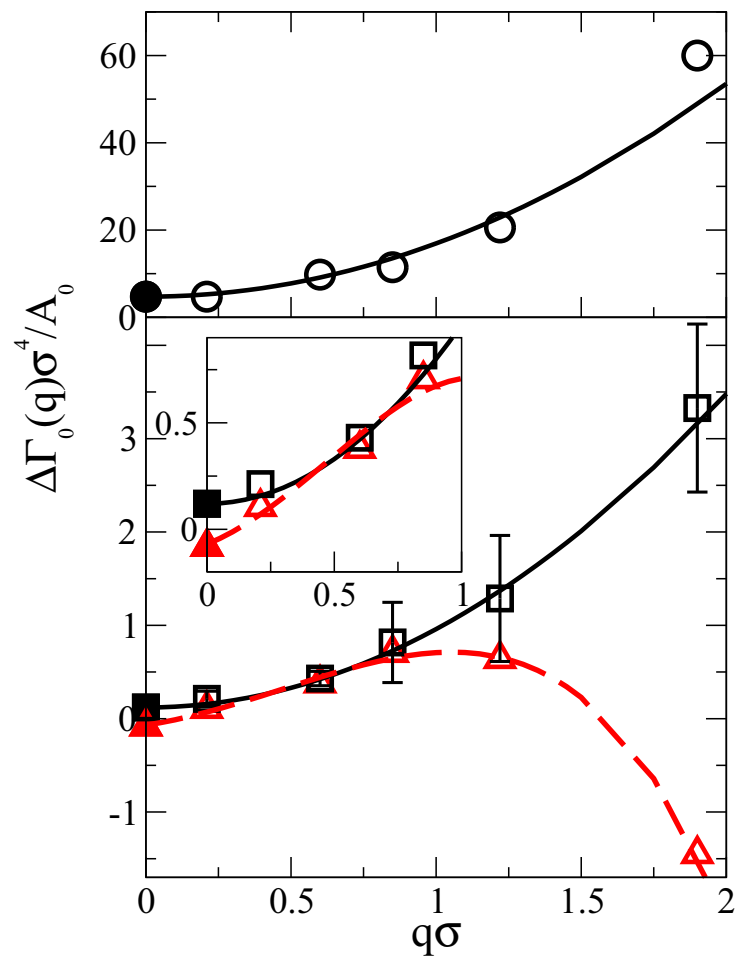

FIG. 4. (Color online) The adimensional $\Delta \Gamma_{0}(q) / A_{0}=$ $\Delta \Gamma\left(q, \xi_{\text {IS }}\right) /\left[A_{0} \exp \left(-\lambda \xi_{\text {IS }}\right)\right]$, with $\lambda \sigma=1.55$, versus $q$. The values are for the largest lateral sizes of the simulation box, $L_{x}=31 \sigma$. The empty symbols show the $\Delta \Gamma_{0}(q)$ simulation results for the three values of $\epsilon_{\mathrm{sf}}: \epsilon_{\mathrm{sf}}=1.3 \epsilon$ (top); $\epsilon_{\mathrm{sf}}=0.81 \epsilon$ (bottom, black squares); and $\epsilon_{\mathrm{sf}}=0.77 \epsilon$ (bottom, red triangles up). The full symbols are the theoretical prediction for $q=0, \Delta \Gamma_{0}(q=0) / A_{0}=\lambda^{2} \beta \Phi_{0}$. The full lines are the better fits to quadratic function: $\lambda^{2} \beta \Phi_{0}+\Delta \gamma_{\mathrm{FJ}} q^{2}$. The dashed line is a guide to the eye.

For the two systems at complete wetting, and within our accuracy, we obtain a parabolic shape $\Delta \Gamma(q) /\left(\beta A_{0}\right) \approx$ $\lambda^{2} \Phi_{0}+q^{2} \Delta \gamma_{\mathrm{FJ}}$, with the Fisher-Jin amplitude of the surface tension wall-enhancement as the only free parameter: $\beta \Delta \gamma_{\mathrm{FJ}} \sigma^{2}=10 \pm 2.5$ for $\epsilon_{\mathrm{sf}} / \epsilon=1.3$ and $\Delta \gamma_{\mathrm{FJ}} \sigma^{2}=0.83 \pm$ 0.04 for $\epsilon_{\mathrm{sf}} / \epsilon=0.81$. These values for the wall effect on the surface tension of the film are very relevant for the liquid films simulated here, since they represent a significant modification, $\Delta \gamma_{\mathrm{FJ}}(\bar{\xi})$, over the surface tension for the free-liquid interface $\beta \gamma_{\mathrm{LV}} \sigma^{2}=0.66$ assumed in the classical mesoscopic Hamiltonian Eq. (2). On the other hand, the nonlocal effects, neglected both in the classical and in the local Fisher-Jin Hamiltonian models, are represented by the $q$ dependence in $\gamma^{\mathrm{LV}}(q) \approx$ $\gamma_{\mathrm{LV}}+\kappa_{\mathrm{LV}} q^{2}+\cdots$, and in $\Delta \gamma_{0}(q) \approx \Delta \gamma_{\mathrm{FJ}}+\Delta \kappa q^{2}+\cdots$. From our ISM results we obtain that the free-surface bending term $\beta \kappa_{\mathrm{LV}} \approx 0.5$ is important to predict the film-thickness fluctuations observed in our computer simulations. However, the possible wall-enhancement $\Delta \kappa$ is much smaller, $0 \lesssim$ $\beta \Delta \kappa \lesssim 0.2$ for $\epsilon_{\mathrm{sf}} / \epsilon=1.3$ and $0 \lesssim \beta \Delta \kappa \lesssim 0.03$ for $\epsilon_{\mathrm{sf}} / \epsilon=$ 0.81 .

Therefore, mean-square fluctuations $\left\langle\left|\hat{\xi}_{q}\right|^{2}\right\rangle$ of the film, observed in our canonical Monte Carlo simulations for all the mean film thickness $\xi_{\text {IS }}$, may be described in terms of:

(i) The fluctuations in the free-liquid surface, given by $\gamma^{\mathrm{LV}}(q) \approx \gamma_{\mathrm{LV}}+\kappa_{\mathrm{LV}} q^{2}$, with the surface tension $\beta \gamma_{\mathrm{LV}} \sigma^{2}=$
0.66 , and the ISM value for the surface bending modulus $\beta \kappa_{\mathrm{LV}}=0.5$.

(ii) The value of $\Phi_{0}\left(\epsilon_{\mathrm{sf}}\right)$ and the correlation length of the liquid bulk, $\lambda^{-1}$, to get the effective potential of the substrate on the film [Eq. (4)].

(iii) The value of $\Delta \gamma_{\mathrm{FJ}}\left(\epsilon_{\mathrm{sf}}\right)$, to describe the wall on the effective surface tension of the film edge, and possibly a small bending term $\Delta \kappa\left(\epsilon_{\mathrm{sf}}\right)$.

The nonlocal theory of Parry et al. [9,12], based on the gradient expansion for the density-functional free energy of the fluid, gives the asymptotic form

$$
\frac{\Delta \Gamma(q, \xi)}{\beta A_{0}}=\Phi_{0}\left(\lambda^{2}+q^{2}\right) e^{-\lambda \xi_{\mathrm{IS}}}
$$

i.e., $\Delta \gamma_{\mathrm{FJ}}=\Phi_{0}$. Here we have taken $\Phi_{0}$ and $\Delta \gamma_{\mathrm{FJ}}$ as independent free parameters, to describe the effective potential for the mean film thickness $\Phi(\bar{\xi})$ with $\Phi_{0}$, and the film thickness fluctuations with $\Delta \gamma_{\mathrm{FJ}}$. They are free parameters used to describe the effects of the wall on the fluctuating liquid film, and we find that both for $\epsilon_{\mathrm{sf}} / \epsilon=1.3$ (deep in the complete wetting regime) and $\epsilon_{\mathrm{sf}} / \epsilon=0.81$ (at the verge of the wetting transition), our values for $\Delta \gamma_{\mathrm{FJ}}$ are clearly larger than the prediction Eq. (6), $\Delta \gamma_{\mathrm{FJ}} / \Phi_{0} \approx 5$ and 17, for $\epsilon_{\mathrm{sf}} / \epsilon=1.3$ and 0.81 , respectively.

From Eq. (3) there is a typical distance $\ell=\sqrt{\Delta \gamma_{0} / \Phi_{0}^{\prime \prime}}$ that scales wavevector $q$ to give the fluctuation damping by the wall. For $q \ell \ll 1$ the dominant effect comes from the wall-fluid potential $\Phi^{\prime \prime}(\bar{\xi})$, while for $q \ell \gg 1$ the most relevant effect comes from the enhancement of the surface tension at the edge of the film $\Delta \gamma(q, \bar{\xi})$. In the square-gradient density functional used by Parry et al. [9,12] the only length scale in the model is given by the bulk correlation length $\lambda^{-1}$, and the prediction Eq. (6) amounts to take precisely $\ell \lambda=1$, since the natural dependence with $q$ within that density-functional model comes as $\lambda^{2}+q^{2}$. However, in a molecular description of the liquid films, far form the critical point, it is clear that the bulk correlation length is not the only relevant length scale. It is not surprising to find larger value, $l \lambda \simeq 1.4-4$, in our realistic model, reflecting the enhancement of the surface tension beyond the direct effect of $\Phi(\bar{\xi})$.

Finally, the fluctuations of the film for $\epsilon_{\mathrm{sf}} / \epsilon=0.77$, in the partial wetting regime, break clearly the form of Eq. (5). The extrapolation of the fluctuation results to $q=0$ is still valid, but that value is negative. The films sampled and presented in Fig. 2 would be unstable in a macroscopic system, and the spring constants $\Gamma(q)$ become negative for low- $q$ modes. The finite size of the simulations boxes stabilize the films, since even with the lowest accessible wavevector ( $q=2 \pi / L_{x}=0.21 / \sigma$ in the larger systems), the positive contribution, $q^{2}\left[\gamma^{\mathrm{LV}}(q)+\Delta \gamma(q, \bar{\xi})\right]$, compensates the negative $\Phi^{\prime \prime}(\bar{\xi})$, while the $q=0$ components of the fluctuations are eliminated by the canonical ensemble constraint on the total number of molecules. Nevertheless, the nonanalytic dependence $\Delta \Gamma_{0}(q)-\Delta \Gamma_{0}(0) \sim q$ (rather than $q^{2}$ ) for low $q$, and the strongly negative value of $\Delta \Gamma_{0}(q)$ for $q=1.9 / \sigma$ indicate the formation of large domains with relatively sharp boundaries that signal the instability of the macroscopic film. A similar behavior was observed, e.g., on Newton black films, 
which behave as a wetting system [30], due to the existence of domains (droplets) with different sizes and widths.

\section{CONCLUDING REMARKS}

In this work we have obtained a complete quantitative characterization of the mesoscopic effective Hamiltonian $\mathcal{H}[\xi]$ that describes the harmonic (quadratic order) fluctuations of a Lennard-Jones liquid film adsorbed on a planar wall. We use canonical and constrained grand-canonical Monte Carlo simulations, and the ISM to get the fluctuating shape of the outer edge of the liquid film. Our results give directly the effective potential of the wall on the liquid film $\Phi(\bar{\xi})$ and the enhancement of the surface tension near the wall $\Delta \Gamma(q, \bar{\xi})$. The latter, neglected by the classical effective Hamiltonian, is clearly as important as $\Phi(\bar{\xi})$ for film fluctuations with wavelength of $10 \mathrm{~nm}$ or shorter. Both $\Phi(\bar{\xi})$ and $\Delta \Gamma(q, \bar{\xi})$ are observed to decay with the correlation length of the liquid bulk, from very thin films of just one or two molecular layers. However, that simple behavior is only observed in simulations done in large enough systems. Previous results, showing a more complex oscillatory structure of $\Delta \Gamma(q, \bar{\xi})$, similar to that of the density profiles, were due to finite-size effects and disappear in our larger simulated systems. The bonus is that the whole dependence of $\mathcal{H}[\xi]$ with the mean film thickness $\bar{\xi}$ is given by the inverse correlation length of bulk liquid $\lambda$ and two amplitudes $\Phi_{0}$ and $\Delta \gamma_{\mathrm{FJ}}$ that depend on the wall-fluid interactions. The values for these two amplitudes may be obtained from computer simulations with less than five molecular layers, and the exponential decay may be safely taken to cover the full range of thicker films.

Together with the correlative ISM analysis for the free liquid-vapor interface, we get a full characterization of $\mathcal{H}[\xi]$, which follows closely, but not completely, the predictions of the nonlocal theory based on a density-functional analysis with the Landau-Ginsburg-Wilson (LGW) free energy $[9,12]$. The first apparent discrepancy, the lack of a second exponential term $\sim e^{-2 \lambda \bar{\xi}}$ in $\Phi(\bar{\xi})$, comes probably from the difference between the bare potential predicted by any mean-field theory and the results of the simulations, which are partially dressed by all the fluctuations within the box size. The second difference with the theoretical LGW prediction is that in our simulations we find that the characteristic length $\sqrt{\Delta \gamma(q, \bar{\xi}) / \Phi(\bar{\xi})}$ is clearly larger than $\lambda^{-1}$. That could be a limitation of the theoretical model, since the LGW density functional lacks of any molecular length other than $\lambda^{-1}$, but very probably it does not affect the qualitative analysis of the problem. The third discrepancy comes in the surface-bending term, the $q^{2}$ coefficient in the expansion of $\gamma(q)$, neglected by the local, nonclassical theory of Fisher and Jin [13]. Our results indicate that this term is small for the wall enhancement term, $\Delta \gamma(q, \bar{\xi}) \approx \Delta \gamma_{\mathrm{FJ}} e^{-\lambda \bar{\xi}}$, but a positive surface-bending rigidity $\kappa_{\mathrm{LV}}$ is crucial to describe the fluctuations of the free-liquid surface. The nonlocal theory of Parry and coworkers [12] finds that a bending term appears from the nonlocal analysis of the LGW, but it has negative value and $\gamma^{\mathrm{LV}}(q)$ decays to zero at large $q$. Similar shape is observed in our computer simulations if we use the Gibbs Surface definition of the film thickness, as shown in the Appendix. Formally, that definition for the film thickness, or the LGW theoretical results for $\gamma^{\mathrm{LV}}(q)$, may always be used in the surface Hamiltonian Eqs. (1) and (3), but they would give a rather counterintuitive view of adsorbed film, with very large surface fluctuations at small wavelengths. However, with different definitions for the effective surface bending from the same model, positive values appear [31], and it is still not clear what would be the precise form of the nonlocal effective $\mathcal{H}[\xi]$ that emanates from the LGW density functional.

In any case, for possible applications to the study of fluctuating adsorbed films at mesoscopic scale, the Hamiltonian $\mathcal{H}[\xi]$ Eq. (1) is technically equivalent to that of nonclassical densityfunctional theories. What we have shown here is that such Hamiltonians may be validated in computer simulations and quantitatively characterized from the molecular interactions. The route is open to link the molecular structure of thin films, with just a few molecular layers, with the renormalization group analysis of the film fluctuation at very large scales. The realistic but simple model used here has to be regarded as a proof of principle for the method. The inclusion of long-range dispersion forces (not-truncated LJ interactions) would provide an even more realistic view of the system and add some new qualitative elements. These problems had already been partially addressed with the ISM analysis of computer simulation for the effects of the wall-fluid [26] and in the liquid-vapor interface [24], and the route to their study within the methodology used here appears to be open. The method could also be applied to rough substrates [32], allowing for a nonzero mean amplitude of the film thickness $\left\langle\hat{\xi}_{q}\right\rangle$ following the corrugations of the substrate, as well as to more complex fluids and mixtures, to address the complex phenomenology of these important interfacial systems.

\section{ACKNOWLEDGMENTS}

We are grateful to A. O. Parry for most useful comments on this work, and we acknowledge the support of the Spanish Ministry of Science and Innovation (Grants No. FIS201022047-C05 and No. FIS2013-47350-C5).

\section{APPENDIX: MODEL, METHODS, AND GIBBS SURFACE RESULTS}

We simulate a LJ model fluid, with the usual parameters $\epsilon$ and $\sigma$ for the energy and length scales. The interactions are truncated at a cutoff distance of $2.5 \sigma$ and all our simulations are done at the same temperature, $k_{\mathrm{b}} T / \epsilon \equiv(\beta \epsilon)^{-1}=0.75$. The structureless substrate is represented by an external potential $V_{\text {sf }}(z)$ acting on the fluid particles, that depends only on their distance to the substrate plane. We take

$$
\begin{aligned}
\frac{V_{\mathrm{sf}}(z)}{8 \pi \epsilon_{\mathrm{sf}} \sigma_{\mathrm{sf}} \rho_{\text {lay }}}= & \sum_{i=1}^{3}\left\{\left[\frac{1}{10}\left(\frac{\sigma_{\mathrm{sf}}}{\left(z-z_{i}\right)}\right)^{10}-\frac{1}{4}\left(\frac{\sigma_{\mathrm{sf}}}{\left(z-z_{i}\right)}\right)^{4}\right]\right. \\
& \left.-\left[\frac{1}{10}\left(\frac{\sigma_{\mathrm{sf}}}{r_{c}}\right)^{10}-\frac{1}{4}\left(\frac{\sigma_{\mathrm{sf}}}{r_{c}}\right)^{4}\right]\right\},
\end{aligned}
$$

to represent the sum over three truncated 4-10 potential layers, each given by the integral over a homogeneous planar density $\rho_{\text {lay }}=1.143 \sigma^{2}$ of LJ interactions with $\sigma_{\mathrm{sf}}=0.912 \sigma$, cut at $\mathrm{r}_{c}=2.5 \sigma_{\mathrm{sf}}$. The layers are at positions $z_{i}=0.0,-0.65 \sigma$, 
and $-1.30 \sigma$, and the strength of the solid-fluid interaction $\epsilon_{\mathrm{sf}}$ is used to explore different wetting regimes at the same temperature. Opposite to the substrate, the simulation cells are closed by a purely repulsive potential at $z=L_{z}=50.0 \sigma$, and square periodic boundary conditions with length $L_{\mathrm{x}}$ are used on the $X$ and $Y$ directions. We compare the results for the lateral size of $L_{x}=10.457 \sigma$ used in our previous work [22] for $\epsilon_{\mathrm{sf}}=1.3$ (and similar size $L_{x}=10 \sigma$ for the new simulations with $\epsilon_{\mathrm{sf}}=0.81$ and 0.77 ) with those for much larger boxes, $L_{x}=31.371 \sigma$ for $\epsilon_{\mathrm{sf}}=1.3$, and $L_{x}=30 \sigma$ for $\epsilon_{\mathrm{sf}}=0.81$ and 0.77. Adsorbed films of different thickness were simulated using $N=160$ to $900 \mathrm{LJ}$ particles for $\epsilon_{\mathrm{sf}}=0.81 \epsilon$, and $0.77 \epsilon$ and $N=150$ to 2000 for the smallest cell size, and $N=1080$ to $4230 \mathrm{LJ}$ particles for $\epsilon_{\mathrm{sf}}=0.81 \epsilon$, and $0.77 \epsilon$ and $N=1350$ to 6750 for the largest cell size.

The effective wall potential on the film thickness was obtained from MC simulations in the grand-canonical ensemble but with a shifting restriction on the total number of particles, to get overlapping probability distributions that could be merged into a single function $\Phi(\xi)$. This method [28,29] provides a good statistical sampling over the whole range of film thickness presented in Fig. 1.

The fluctuations at the edge of the wetting films are explored in a set of canonical MC simulations, with the mean film thickness controlled by the total number of particles. For each system $10^{4}$ configurations, separated by $2000 \mathrm{MC}$ steps, were taken after $20 \times 10^{6} \mathrm{MC}$ steps for equilibration. We used two different methods, the GS and the ISM, to determine the function $\xi(\mathbf{R})=\sum_{\mathbf{q}} \hat{\xi}_{\mathbf{q}} \exp (\mathbf{i q} \cdot \mathbf{R})$ that describes the film thickness at each point $\mathbf{R}$ on the substrate plane. The simplest GS representation relates the thickness $\xi^{\mathrm{GS}}(\mathbf{R})$ to the number of particles in the system whose projection on the substrate happen to be around position $\mathbf{R}$, irrespective of their $z$ coordinate. That number of particles per unit area is transformed in a local film thickness assuming that the surface $z=\xi^{\mathrm{GS}}(\mathbf{R})$ separates a uniform density of the liquid phase from a uniform density of the vapor phase. Therefore, the $q \neq 0$ Fourier components are directly given from the position of the particles [11], as

$$
\hat{\xi}_{q}^{\mathrm{GS}}=\frac{1}{A_{0} \Delta \rho_{\mathrm{LV}}} \sum_{j=1}^{N} e^{i \mathbf{q} \cdot \mathbf{R}_{j}},
$$

and the $q=0$ component is the usual Gibbs dividing plane that gives the mean film thickness as $\hat{\xi}_{0}=N /\left(\Delta \rho_{\mathrm{LV}} A_{0}\right)-z_{0}$, where $\Delta \rho_{\mathrm{LV}}$ is the density difference between the bulk phases, $A_{0}=L_{x}^{2}$ is the substrate area, and $z_{0}$ is a nominal position for the effective wall of the substrate, irrelevant for the $q \neq 0$ components. The drawback of this simple definition for $\xi(\mathbf{R})$ is that it assumes as thickness fluctuations any instantaneous change in the local density of the liquid film; therefore, for thick films $\left\langle\left|\hat{\xi}_{q}^{\mathrm{GS}}\right|^{2}\right\rangle$ does not decay as $\sim 1 /\left(q^{2} \gamma_{\mathrm{LV}}\right)$ for large $q$ [as predicted by Eq. (2)], but it goes to a constant high- $q$ limit related to the bulk liquid compressibility. From that effect, the GS result for $\gamma^{\mathrm{LV}}(q)$ decays to zero for large $q$, as shown in the inset of Fig. 5, and the mesoscopic Hamiltonian Eq. (1), with this $\gamma^{\mathrm{LV}}(q)$ in Eq. (3), would predict very large amplitudes for fluctuations with very small wavelength.

In this work we have used the ISM as a computationally more costly, but qualitatively more accurate, description of

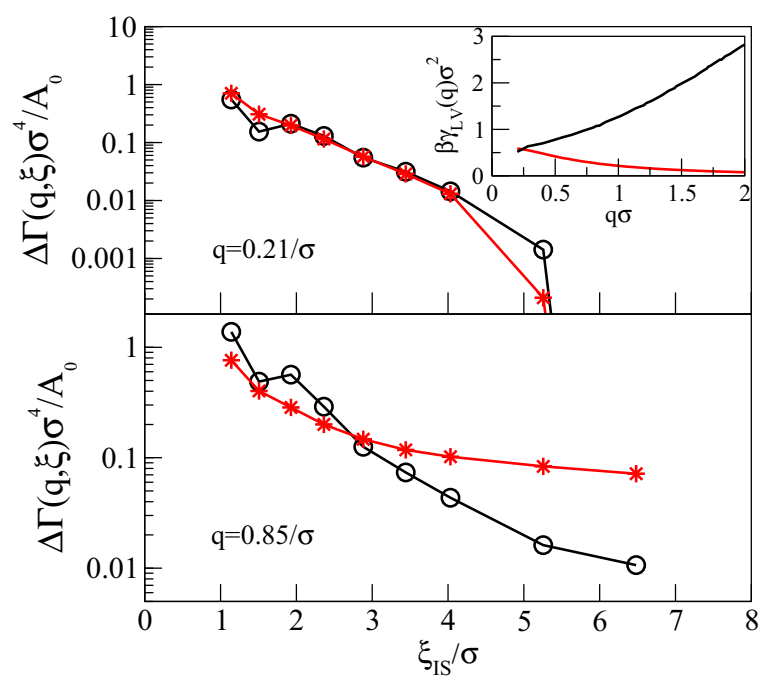

FIG. 5. (Color online) The function $\Delta \Gamma\left(q, \xi_{\text {IS }}\right) \sigma^{4} / A_{0}$ (as in Fig. 2, but in logarithmic scale), for $L_{x}=31 \sigma, \epsilon_{\mathrm{sf}}=1.3 \epsilon$, and two values of the wavevector $q \sigma=0.21$ (top) and $q \sigma=0.85$ (bottom). Black circles give the ISM results and gray (on line red) starts symbols show the results of the Gibbs surface (GS) film thickness; the lines are only to guide the eye. The inset shows the surface tension function $\gamma^{\mathrm{LV}}(q)$ for the liquid-vapor interface, ISM black line and GS gray (on line red) line.

the film thickness. This method is based on the identification of the particles at the boundary of the percolation cluster formed by the liquid film and by a smooth interpolation of the mathematical surface $z=\xi^{\mathrm{IS}}(\mathbf{R})$ going through the most external layers of molecules in the liquid film. Both the percolation analysis and the interpolation scheme have been optimized in the study of the free-liquid surface [33], and we keep here the same parameters and operational procedure that has been described in previous works [22,23].

The ISM results are presented in Figs. 2-4, and they provide the main body of our work. Figure 5 presents the comparison with the GS results only for two cases, $q \sigma=0.21$ and 0.85 , in the largest simulation box, $L_{x}=31 \sigma$, for $\epsilon_{\mathrm{sf}}=1.3 \epsilon$. The decay of $\Delta \Gamma(q, \xi)$ with the mean thickness of the film is presented in logarithmic scale, instead of the linear scale shown in Fig. 2. It is clear that the GS definition for the film thickness is accurate and consistent with the ISM result for the lower $q$, as it is in the $q=0$ limit used to get $\Phi(\xi)$. However, for $q \sigma=0.85$ the decay of $\Delta \Gamma^{\mathrm{GS}}(q, \xi)$ for the largest film thickness differs qualitatively from the ISM result. The exponential decay of the ISM results may be followed down for three decades, while the GS goes to a much larger asymptotic value, incompatible with the theoretical prediction Eq. (6). The amplitude of the exponential decay, avoiding that asymptotic limit of the GS result, would have much larger error bars than the ISM results presented in Fig. 4, and it would be difficult to discern the functional form of $\Delta \Gamma_{0}^{\mathrm{GS}}(q)$ even for its low $q$ expansion.

Therefore, the ISM is worth its much higher computational cost, because it provides a definition for the local thickness of the adsorbed film that is not affected by compressibility fluctuations within the liquid film, that spoil the GS results in thick films for large $q$. Notice that the main effect of the 
spurious inclusion of these fluctuations in $\left\langle\left|\hat{\xi}_{q}^{\mathrm{GS}}\right|^{2}\right\rangle$ appears in the free liquid-vapor interface, with the qualitative difference between the ISM and GS results for $\gamma^{\mathrm{LV}}(q)$ shown in the inset of Fig. 5. The raising shape of the $\operatorname{ISM} \gamma^{\mathrm{LV}}(q)$ provides a natural molecular top to the spectrum of capillary fluctuations, while the use of the GS results in the mesoscopic surface Hamiltonian Eq. (1) would give a rather hectic image of the liquid surface, with very large nominal corrugations at very low wavelengths. Certainly, there is not a unique definition for
$\xi(\mathbf{R})$, the mesoscopic description of the adsorbed film would always require some operational definition of its thickness in terms of the molecular positions, and each definition would give different functions $\gamma^{\mathrm{LV}}(q)$ and $\Delta \Gamma(q, \xi)$ within the same formal description [Eq. (3)]. The advantage of a better choice to define $\xi(\mathbf{R})$ is not of formal consistency, but rather of a natural physical interpretation of the mesoscopic variables. Unfortunately, the GS [Eq. (A2)] is a property more akin to experimental measurements than is ISM.
[1] P.-G. de Gennes, F. Brochard-Wyard, and D. Quéré, Capillarity and Wetting Phenomena (Springer, Berlin, 2003).

[2] M. Rauscher and S. Dietrich, Wetting phenomena in nanofluidics, Ann. Rev. Mater. Res. 38, 143 (2008).

[3] J. R. Henderson, Statistical mechanics of the disjoining pressure of a planar film, Phys. Rev. E 72, 051602 (2005).

[4] D. Bonn, J. Eggers, J. Indekeu, J. Meunier, and E. Rolley, Wetting and spreading, Rev. Mod. Phys. 81, 739 (2009).

[5] F. P. Buff, R. A. Lovett, and F. H. Stillinger, Interfacial density profile for fluids in the critical region, Phys. Rev. Lett. 15, 621 (1965).

[6] R. Evans, The nature of the liquid-vapour interface and other topics in the statistical mechanics of non-uniform, classical fluids, Advan. Phys. 28, 143 (1979).

[7] J. R. Percus, in Fluid Interfacial Phenomena, edited by C. A. Croxton (John Wiley, New York, 1986), pp. 1-44.

[8] A. J. Jin and M. E. Fisher, Stiffness instability in short-range critical wetting, Phys. Rev. B. 48, 2642 (1993).

[9] A. O. Parry, Three-dimensional wetting revisited, J. Phys.: Condens. Matter 8, 10761 (1996).

[10] E. Chacón and P. Tarazona, Intrinsic profiles beyond the capillary wave theory: A Monte Carlo study, Phys. Rev. Lett. 91, 166103 (2003).

[11] P. Tarazona and E. Chacón, Monte Carlo intrinsic surfaces and density profiles for liquid surfaces, Phys. Rev. B 70, 235407 (2004).

[12] A. O. Parry, C. Rascón, N. R. Bernardino, and J. M. RomeroEnrique, Non-locality and short-range wetting phenomena, Phys. Rev. Lett. 100, 136105 (2008).

[13] M. E. Fisher and A. J. Jin, Is short-range "critical" wetting a first-order transition?, Phys. Rev. Lett. 69, 792 (1992).

[14] A. O. Parry, J. M. Romero-Enrique, and A. Lazarides, Nonlocality and short-range wetting phenomena, Phys. Rev. Lett. 93, 086104 (2004).

[15] Y. Hennequin, D. G. A. L. Aarts, J. O. Indekeu, H. N. W. Lekkerkerker, and D. Bonn, Fluctuation forces and wetting layers in colloid-polymer mixtures, Phys. Rev. Lett. 100, 178305 (2008).

[16] M. Fukuto, O. Gang, K. J. Alvine, and P. S. Pershan, Capillary wave fluctuations and intrinsic widths of coupled fluid-fluid interfaces: An x-ray scattering study of a wetting film on bulk liquid, Phys. Rev. E 74, 031607 (2006).

[17] P. Huber, O. Shpyrko, P. S. Pershan, B. Ocko, E. DiMasi, and M. Deutsch, Short-range wetting at liquid gallium-bismuth alloy surfaces: X-ray measurements and square-gradient theory, Phys. Rev. B 68, 085409 (2003).

[18] L. Pang, D. P. Landau, and K. Binder, Simulation evidence for nonlocal interface models: Two correlation lengths describe complete wetting, Phys. Rev. Lett. 106, 236102 (2011).

[19] A. Milchev, A. Milchev, and K. Binder, Nanodroplets on a solid plane: wetting and spreading in a Monte Carlo simulation, Comput. Phys. Commun. 146, 38 (2002).

[20] P. Bryk and K. Binder, Non-mean-field behavior of critical wetting transition for short-range forces, Phys. Rev. E 88, 030401(R) (2013).

[21] E. M. Fernández, E. Chacón, P. Tarazona, A. O. Parry, and C. Rascón, Intrinsic fluid interfaces and nonlocality, Phys. Rev. Lett. 111, 096104 (2013).

[22] E. M. Fernández, E. Chacón, and P. Tarazona, Capillary wave spectrum at adsorbed liquid films, Phys. Rev. B 86, 085401 (2012).

[23] E. M. Fernández, E. Chacón, and P. Tarazona, Thickness and fluctuations of free and adsorbed liquid films, Phys. Rev. B 84, 205435 (2011).

[24] E. Chacón, E. M. Fernández, and P. Tarazona, Effect of dispersion forces on the capillary-wave fluctuations of liquid surfaces, Phys. Rev. E 89, 042406 (2014).

[25] M. Napiórkowski and S. Dietrich, Structure of the effective Hamiltonian for liquid-vapor interfaces, Phys. Rev. E 47, 1836 (1993).

[26] L. G. MacDowell, J. Benet, and N. A. Katcho, Capillary fluctuations and film-height-dependent surface tension of an adsorbed liquid film, Phys. Rev. Lett. 111, 047802 (2013).

[27] N. R. Bernardino, A. O. Parry, C. Rascón, and J. M. RomeroEnrique, Derivation of a non-local interfacial model for 3D wetting in an external field, J. Phys.: Condens. Matter 21, 465105 (2009).

[28] L. G. MacDowell and M. Müller, Observation of autophobic dewetting on polymer brushes from computer simulation, J. Phys.: Condens. Matter 17, S3523 (2005).

[29] L. G. MacDowell and M. Müller, Adsorption of polymers on a brush: Tuning the order of the wetting phase transition, J. Chem. Phys. 124, 084907 (2006).

[30] P. Tarazona, H. Martínez, E. Chacón, and F. Bresme, Newton black films as wetting systems, Phys. Rev. B 85, 085402 (2012).

[31] A. O. Parry, C. Rascón, G. Willis, and R. Evans, Parr correlation functions and the wavevector-dependent surface tension in a simple density functional treatment of the liquid-vapour interface, J. Phys.: Condens. Matter 35, 355008 (2014).

[32] S. Dietrich, M. N. Popescu, and M. Rauscher, Wetting on structured substrates, J. Phys.: Condens. Matter 17, S577 (2005).

[33] E. Chacón, E. M. Fernández, D. Duque, R. Delgado-Buscalioni, and $\mathrm{P}$. Tarazona, Comparative study of the surface layer density of liquid surfaces, Phys. Rev. B 80, 195403 (2009). 